\title{
AN INEQUALITY FOR THE GIBBS MEAN NUMBER OF CLUSTERS
}

\author{
CAMILLO CAMMAROTA
}

\begin{abstract}
The number of percolation clusters for configurations of the Ising model at zero external field and ferromagnetic first neighbors interaction on a general finite graph is considered. The mean number of clusters with respect to the Gibbs measure at any inverse temperature $\beta$ is proved to be smaller or equal than the one at $\beta=0$.
\end{abstract}

2000 Mathematics Subject Classification 82B43, 82B20, 60 K35.

Keywords: cluster, percolation, Gibbs measure, temperature, group.

\section{INTRODUCTION}

Let $G=(V, E)$ be a finite connected graph, of vertexes set $V$ and edges set $E$. The Gibbs measure on $G$ is the probability measure $\mu_{\beta}$ on $\Sigma=\{-1,1\}^{V}$ of weight

$$
e^{\beta \sum_{\{i, j\} \in E} \sigma(i) \sigma(j)}, \quad \sigma \in \Sigma
$$

We denote by $\mathbb{E}_{\mu_{\beta}}^{G}$ the mean with respect to this measure. The measure $\mu_{\beta}$ describes an Ising model with spin variables on vertexes, ferromagnetic interaction on edges, zero external field and inverse temperature $\beta$. Given any configuration $\sigma \in \Sigma$, the $+(-)$ clusters of $\sigma$ are the maximal connected components of $\sigma^{-1}(+1)\left(\sigma^{-1}(-1)\right)$. We denote by $C^{+(-)}(\sigma)$ the set of $+(-)$ clusters of $\sigma$; the number of $+(-)$ clusters $N^{+(-)}(\sigma)$ is the cardinality of $C^{+(-)}(\sigma)$, also denoted $\left|C^{+(-)}(\sigma)\right|$. The number of clusters of $\sigma$, denoted $N(\sigma)$, is defined as $N(\sigma)=N^{+}(\sigma)+N^{-}(\sigma)$ and obviously one has by symmetry

$$
\mathbb{E}_{\mu_{\beta}}^{G}\left(N^{+}\right)=\mathbb{E}_{\mu_{\beta}}^{G}\left(N^{-}\right)
$$

It is useful to consider the family $\mu_{\beta}, \quad \beta \in[0,+\infty]$. One easily recognizes that $\mu_{0}$ is a Bernoulli measure of parameter $\frac{1}{2}$ and that $\mu_{\infty}=\frac{1}{2} \delta_{+}+\frac{1}{2} \delta_{-}$, where $\delta_{+}\left(\delta_{-}\right)$is the point mass concentrated on the constant $+(-)$ configuration. Consequently one has $\mathbb{E}_{\mu_{\infty}}^{G}(N)=1$, while the value of $\mathbb{E}_{\mu_{0}}^{G}(N)$ depends on graph.

The random variable number of clusters plays a central role in percolation theory, and in case of Bernoulli percolation it has been extensively studied (for a general reference see [8]). We are interested in monotonicity properties with respect to $\beta$ of the mean number of 
clusters $\mathbb{E}_{\mu_{\beta}}^{G}(N)$, as a particular case of the general problem of monotonicity in $\beta$ for variables used in percolation theory. As an example, it is an open problem to extend the coexistence of + and - infinite clusters proved in the cubic lattice for the Ising model at $\beta$ close to 0 [6] to a larger range of temperatures.

The Gibbs measure is FKG for fixed temperature (for instance see [9]), but there is not a satisfactory notion of stochastic ordering with respect to temperature. To circumvent this problem one method is to represent the measure on spins by means of a measure on edges configurations. A widespread approach is the random cluster model given by the Fortuin-Kasteleyn representation. For a review we refer to [9]. Since random cluster measures are stochastically ordered in temperature, it is enough to prove that a particular observable in edge representation is monotonic in the partial ordering of these configurations. Using this method some observables related to percolation can be proved to have monotonicity properties $[4,7]$.

However other approaches have been used, and in particular we refer to the group representation of Ising model, for which a basic reference is [10]. Combining this representation and the notion of stochastic order has revealed fruitful. In particular some monotonicity properties of local observables can be re obtained and new ones are proved $[2$, 5]. The main idea is that the Gibbs measure on spins configurations is represented by a measure on edges configurations conditioned to a group, and that conditioned measures are stochastically ordered.

Despite some evident similarities with the random cluster model, one advantage of this approach is that the measure at $\beta=0$ is a delta mass concentrated on the empty configuration on edges. This feature can be exploited to treat a simpler type of problems, i. e. the inequality between the means at $\beta>0$ and at $\beta=0$. In [3] the mean number of clusters was proved to be smaller at $\beta>0$ than at $\beta=0$, if the graph has degree non greater than 3 . In the present paper we prove an extension of this inequality for any degree.

Proposition 1.1. For any graph $G$ the mean number of clusters with respect to the Gibbs measure at inverse temperature $\beta>0$ is less or equal to the one at $\beta=0$ :

$$
\mathbb{E}_{\mu_{\beta}}^{G}(N) \leq \mathbb{E}_{\mu_{0}}^{G}(N)
$$

The main contribution of the present paper essentially consists in reducing the problem for a general graph $G$ to the one for a graph $G^{\prime}$ with degree not greater than 3 . This is achieved using a vertex resolution procedure, similar to the one used in [11], and conditioning the measure $\mu_{\beta}$ to a suitable configurations subset $\Delta$. Hence the main ingredients of our proof can be summarized by the following equations

$$
\mathbb{E}_{\mu_{\beta}}^{G}(N)=\mathbb{E}_{\mu_{\beta}}^{G^{\prime}}(N \mid \Delta) \leq \mathbb{E}_{\mu_{0}}^{G^{\prime}}(N \mid \Delta)=\mathbb{E}_{\mu_{0}}^{G}(N)
$$



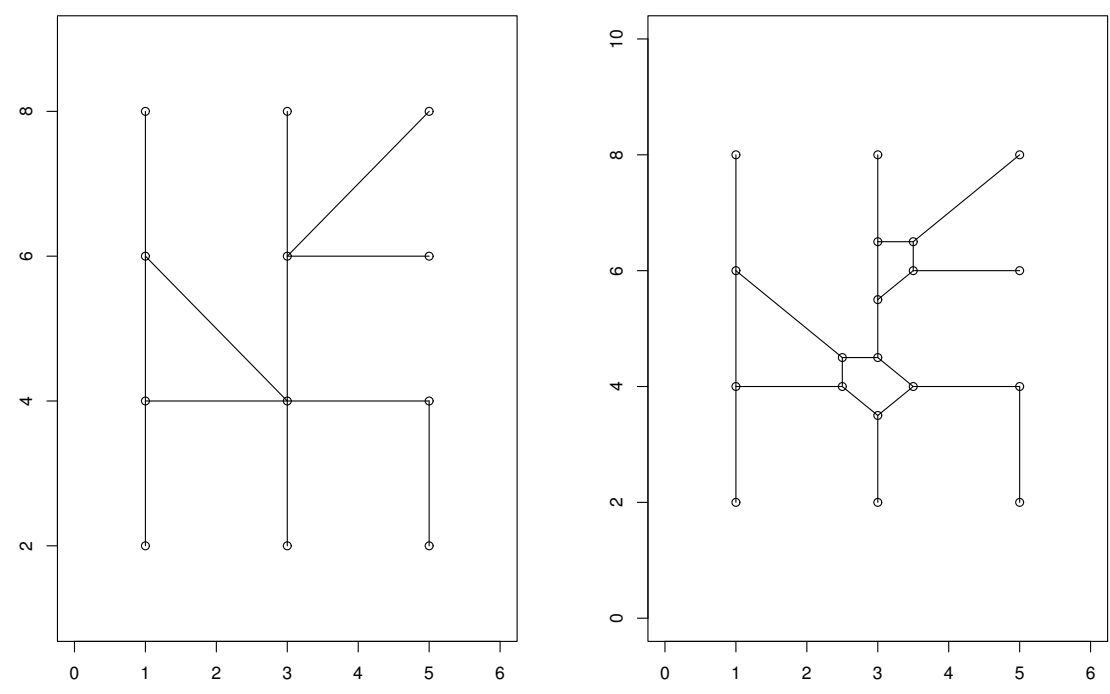

FiguRE 1. Resolution procedure applied to the graph on the left, having one vertex of degree 4 , of coordinates $(3,6)$, and one of degree 5 , of coordinates $(3,4)$; on the right the graph obtained after resolution of these vertexes.

The inequality in the middle is an extension of the one proved in [3] and the external equalities are the result of the resolution procedure. In the present paper we also provide an exposition of the basic elements of the group representation, in order to make the paper self consistent.

\section{VERTEX Resolution}

In this section we prove the following lemma.

Lemma 2.1. There is a graph $G^{\prime}=\left(V^{\prime}, E^{\prime}\right)$ with degree not greater than 3 and a subset $\Delta$ of $\Sigma^{\prime}=\{-1,1\}^{V^{\prime}}$ such that for the number of clusters $N$ the following equation holds

$$
\mathbb{E}_{\mu_{\beta}}^{G}(N)=\mathbb{E}_{\mu_{\beta}}^{G^{\prime}}(N \mid \Delta)
$$

On r.h.s. there is the conditional expectation with respect to $\Delta$, and the Gibbs measure on $G^{\prime}$ is defined as the one on $G$.

Proof. Given a vertex $i \in V$ we denote $\partial i$ the set of the vertexes adjacent to $i$ (first neighbors); the degree of $i$, denoted $\operatorname{deg}(i)$, is the cardinality of $\partial i$. For each $i$ we define a set $B_{i}$, the resolution of the vertex $i$, as follows. If $\operatorname{deg}(i) \leq 3$, we put $B_{i}=\{i\}$; if $\operatorname{deg}(i)>3$, the 
set $B_{i}$ is defined as a replica of $\partial i$, and we denote $j^{\prime}$ the element of $B_{i}$ which corresponds to $j$. The new vertexes set is

$$
V^{\prime}=\cup_{i \in V} B_{i}
$$

In order to define $E^{\prime}$, we first replace the edges $\{i, j\} \in E, j \in \partial i$, by the edges $\left\{j, j^{\prime}\right\}, j \in \partial i$. This defines a set of edges on $V^{\prime}$, called 'conduction' edges and denoted $E_{C}^{\prime}$, which are in one to one correspondence with $E$. We then put in each of the $B_{i}$ 's having cardinality greater than 3 edges such that make it connected; if $B_{i}=\left\{k_{1}, k_{2}, \cdots, k_{n}\right\}$, for instance we choose the edges $\left\{k_{1}, k_{2}\right\}, \cdots,\left\{k_{n}, k_{1}\right\}$, forming a loop on $B_{i}$. We call these 'identification' edges and denote them $E_{C}^{\prime}$. The set $E^{\prime}$ can now be defined as union of conduction edges $E_{C}^{\prime}$ and identification edges $E_{I}^{\prime}$. The vertexes with degree not greater than 3 keep unchanged their degree; the other ones have exactly one conduction edge and two identification edges; hence the degree of $G^{\prime}$ is at most 3. This formal definition can be made more transparent with the help of figure 1 .

For the graph $G^{\prime}$ the Gibbs measure on $\Sigma^{\prime}=\{-1,1\}^{V^{\prime}}$ is formally defined as the one on $G$, and we use the same notation. We define the identification subset $\Delta \subset \Sigma^{\prime}$ as the intersection over $i \in V$ of the identification event which forces all spins in $B_{i}$ to be equal: $\sigma^{\prime}\left(k_{1}\right)=$ $\cdots=\sigma^{\prime}\left(k_{n}\right)$. There is a trivial one to one map between configurations spins in $\Sigma$ and $\Delta$, such that if $\sigma \in \Sigma$ and $\sigma^{\prime} \in \Delta$ one has $\sigma(i)=$ $\sigma^{\prime}(k), k \in B_{i}$. An important consequence of above definitions is that the number of clusters satisfies the equation

$$
N(\sigma)=N\left(\sigma^{\prime}\right)
$$

Furthermore the interactions that define the Gibbs weights differ for a constant depending only on the graph:

$$
\begin{gathered}
\sum_{\{i, j\} \in E^{\prime}} \sigma^{\prime}(i) \sigma^{\prime}(j)=\sum_{\{i, j\} \in E_{C}^{\prime}} \sigma^{\prime}(i) \sigma^{\prime}(j)+\sum_{\{i, j\} \in E_{I}^{\prime}} \sigma^{\prime}(i) \sigma^{\prime}(j) \\
=\sum_{\{i, j\} \in E} \sigma(i) \sigma(j)+\sum_{i \in V, \operatorname{deg}(i)>3}\left|B_{i}\right|
\end{gathered}
$$

where we have used that conduction edges reproduce exactly $E$ and that each identification edge gives a constant contribution of value 1 . This suffices to conclude that conditioned to $\Delta$ the mean number of clusters on $G^{\prime}$ has the same value as the one on $G$.

Remark 1 Identification edges on $B_{i}$ can be chosen in several ways, subject to the condition that they make $B_{i}$ connected and that $\operatorname{deg}(j) \leq$ 3 ; for instance one of edges of the loop above defined can be dropped.

Remark 2 Equation (2) may not be true for other percolation variables. Let us consider the cardinality of the (say + ) cluster containing a fixed vertex; if $W_{k}^{+}\left(\sigma^{\prime}\right)$ denotes the + cluster on $G^{\prime}$ containing the vertex $k \in V^{\prime}$, where $k \in B_{i}$ and $\sigma^{\prime} \in \Delta$, and if $W_{i}^{+}(\sigma)$ denotes the 
corresponding cluster on $G$, they are related by the non trivial relationship

$$
\left|W_{k}^{+}\left(\sigma^{\prime}\right)\right|=\sum_{j \in W_{i}^{+}(\sigma)}\left|B_{j}\right|
$$

\section{Group Representation of COnditioned GibBs measures}

We first discuss the representation of unconditioned Gibbs measures following [2]. Given $\sigma \in \Sigma$ we associate to it the edge configuration $\omega \in \Omega=\{0,1\}^{E}$, defined as $\omega(i, j)=0$ if $\sigma(i)=\sigma(j)$ and $\omega(i, j)=1$ if $\sigma(i) \neq \sigma(j)$. This defines a map $\psi$ from spins to edges configurations; by symmetry one has $\psi(\sigma)=\psi(-\sigma)$. The range of this map, denoted $\Gamma$, can be characterized as the set of edges configurations such that any loop contains an even number of 1's (this was noticed for instance in [7], but with the opposite coding). A next step is to recognize that this set has a group structure, as a subgroup of $\Omega$ [1]. Actually in $\Omega$ there is a product defined as

$$
\omega_{1} \omega_{2}(e)=\omega_{1}(e)+\omega_{2}(e) \quad \bmod 2, \quad e \in E
$$

and the identity is the null element $0(e)=0, e \in E$. We call $\Gamma$ the 'parity' group.

The Gibbs weight can be written as

$$
e^{\beta\left|\omega^{-1}(0)\right|-\beta\left|\omega^{-1}(1)\right|}, \quad \omega \in \Gamma
$$

This is the same as the one of a Bernoulli product measure $\nu_{p}$ of parameters $p$ for the 1's and $1-p$ for the 0 's, where $p=e^{-\beta} /\left(e^{\beta}+e^{-\beta}\right)$, conditioned to $\Gamma$. Since we are interested in variables invariant with respect to total spin flip, their means with respect to the measure $\mu_{\beta}$ can be written in terms of means with respect to the Bernoulli measure $\nu_{p}$ conditioned to the subgroup $\Gamma$. If $X$ denotes such a variable, both as a function of spins configurations and of edges configurations, we have

$$
\mathbb{E}_{\mu_{\beta}}(X)=\mathbb{E}_{\nu_{p}}(X \mid \Gamma)
$$

We now consider the general problem of representing the Bernoulli measure $\nu_{p}$ conditioned to a subgroup $\Theta$. We need some definitions. The cylinder of base $A \subset E$, given $\alpha \in\{0,1\}^{A}$, is

$$
K_{\alpha}^{A}=\{\omega \in \Omega \mid \omega(e)=\alpha(e), e \in A\}
$$

We include the case $A=\emptyset$ putting $K_{\alpha}^{\emptyset}=\Omega$. In particular one has that $K_{0}^{A}$ is a subgroup and $K_{\alpha}^{A}$ is a coset for any $\alpha$. If $X$ is a function on $\Omega$ and $\Theta$ is a subgroup, we define a function on the subsets of $E$

$$
X_{\Theta}(A)=\left|\Theta \cap K_{0}^{A}\right|^{-1} \sum_{\omega \in \Theta \cap K_{0}^{A}} X(\omega)
$$


We also introduce a probability measure $\lambda_{p}^{\Theta}$ on the subsets of $E$

$$
\lambda_{p}^{\Theta}(A)=\nu_{p}(\Theta)^{-1}(1-2 p)^{|A|} p^{|E \backslash A|}\left|\Theta \cap K_{0}^{A}\right|
$$

With these notation we have

Proposition 3.1. [2] The mean of $X$ with respect to the Bernoulli measure $\nu_{p}$ conditioned to a subgroup $\Theta$ can be represented by

$$
\mathbb{E}_{\nu_{p}}(X \mid \Theta)=\sum_{A \subset E} X_{\Theta}(A) \lambda_{p}^{\Theta}(A)
$$

Proof. Denoting for brevity $\omega=\omega^{-1}(1)$ and $\omega^{c}=\omega^{-1}(0)$ and considering $\omega$ as a subset of $E$, one has

$$
\nu_{p}(\omega)=p^{|\omega|}(1-2 p+p)^{\left|\omega^{c}\right|}=\sum_{A \subset \omega^{c}}(1-2 p)^{|A|} p^{|E \backslash A|}
$$

Hence

$$
\sum_{\omega \in \Theta} \nu_{p}(\omega) X(\omega)=\sum_{A \subset E}(1-2 p)^{|A|} p^{|E \backslash A|} \sum_{\omega \in \Theta \cap K_{0}^{A}} X(\omega)
$$

and this concludes the proof.

We can now discuss the representation of conditioned Gibbs measures; in particular we are looking for a suitable representation of $\mathbb{E}_{\mu_{\beta}}^{G^{\prime}}(N \mid \Delta)$. We first notice that the image of $\Delta$ on edges configurations is a subgroup of $\Omega^{\prime}$. In particular it is the cylinder $K_{0}^{E_{I}^{\prime}}$ obtained putting 0 's on identification edges of $E^{\prime}$; we use the same notation $\Delta$ and call it 'identification' group. We shall use the following property. Since the representation of the Gibbs measure requires conditioning to the parity group $\Gamma$, the representation of the Gibbs measure conditioned to identification group $\Delta$ can be achieved conditioning to the intersection of these two groups. We can so write

$$
\mathbb{E}_{\mu_{\beta}}^{G^{\prime}}(N \mid \Delta)=\mathbb{E}_{\nu_{p}}^{G^{\prime}}(N \mid \Gamma \cap \Delta)
$$

Since $\Theta=\Gamma \cap \Delta$ is a group, we can apply eq. (6) and get

$$
\mathbb{E}_{\mu_{\beta}}^{G^{\prime}}(N \mid \Delta)=\sum_{A \subset E} N_{\Theta}(A) \lambda_{p}^{\Theta}(A)
$$

We now remark that for $\beta=0$, i.e. $p=1 / 2$, the corresponding measure $\lambda_{\frac{1}{2}}^{\Theta}$ is a delta mass concentrated on the empty set. Hence

$$
\mathbb{E}_{\mu_{0}}^{G^{\prime}}(N \mid \Delta)=N_{\Theta}(\emptyset)
$$

Hence in order to have

$$
\mathbb{E}_{\mu_{\beta}}^{G^{\prime}}(N \mid \Delta) \leq E_{\mu_{0}}^{G^{\prime}}(N \mid \Delta)
$$

it is sufficient the condition 


$$
N_{\Theta}(A) \leq N_{\Theta}(\emptyset), \quad A \subset E^{\prime}
$$

The next section is devoted to prove this inequality.

\section{A PRUNING TREE ARGUMENT}

We shall prove that the function $N_{\Theta}$ over the subsets of $E^{\prime}$ has the following weak monotonicity property.

Proposition 4.1. For any $A \subset E^{\prime}, A \neq \emptyset$, there is $e \in A$ such that

$$
N_{\Theta}(A) \leq N_{\Theta}(A \backslash e)
$$

One easily recognizes that this property is sufficient to give eq. (11). The case that $\Theta$ is the parity group was considered in [3]. Here we recall that proof, adapting it to the present context.

Proof. Eq. (12) is equivalent to

$$
\left|\Theta \cap K_{0}^{A \backslash e}\right| \sum_{\omega \in \Theta \cap K_{0}^{A}} N(\omega) \leq\left|\Theta \cap K_{0}^{A}\right| \sum_{\omega \in \Theta \cap K_{0}^{A \backslash e}} N(\omega)
$$

We use

$$
\left|\Theta \cap K_{0}^{A \backslash e}\right|=\left|\Theta \cap K_{0}^{A \backslash e e}\right|+\left|\Theta \cap K_{0}^{A \backslash e e}\right|
$$

and

$$
\sum_{\omega \in \Theta \cap K_{0}^{A \backslash e}} N(\omega)=\sum_{\omega \in \Theta \cap K_{0}^{A \backslash e e}} N(\omega)+\sum_{\omega \in \Theta \cap K_{0}^{A \backslash e e}} N(\omega)
$$

and so eq. (13) is equivalent to

$$
\left|\Theta \cap K_{01}^{A \backslash e e}\right| \sum_{\omega \in \Theta \cap K_{\substack{A \backslash e e \\ 00}}^{A}} N(\omega) \leq\left|\Theta \cap K_{00}^{A \backslash e e}\right| \sum_{\omega \in \Theta \cap K_{01}^{A \backslash e e}} N(\omega)
$$

where we have used $\Theta \cap K_{00}^{A \backslash e e}=\Theta \cap K_{0}^{A}$

If the set $\Theta \cap K_{01}^{A \backslash e e}$ is empty, we define zero the sum extended to it and we have by definition

$$
N_{\Theta}(A)=N_{\Theta}(A \backslash e)
$$

Since $\Theta=\Delta \cap \Gamma$, the set $\Theta \cap K_{01}^{A \backslash e e}$ is empty if the value 1 on the edge $e$ violates the identification or the parity condition. The first case happens if $e$ is an identification edge; the second one if $e$ belongs to a loop of $A$, since any loop must contain an even numbers of 1 's.

If the set $\Theta \cap K_{01}^{A \backslash e e}$ is non empty, it is a coset of the group $\Theta \cap K_{0}^{A \backslash e e}$ and so it has the same cardinality. Hence eq. (12) is equivalent to

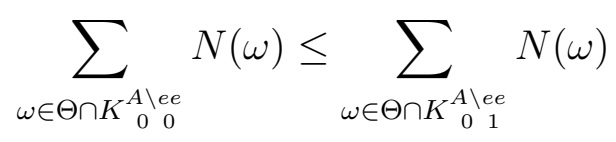

From the previous argument we can now suppose that $A$ does not contains loops, i.e. it is union of disjoint trees and we shall prove that 
pruning any end line $e$ preserves ineq. (12), since eq. (15) holds. It is convenient to rewrite this equation in spins language, getting

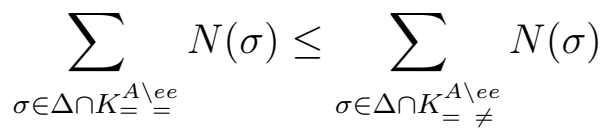

where the set $K_{=\neq}^{A \backslash e e}$ is the spin version of $K_{0}^{A \backslash e e}$. If $e=\{i, j\}$ where $j$ is an endpoint of the tree $T \cup\{j\}$ of $A$, in order to get eq. (15) it is sufficient

$$
N\left(\sigma_{++}^{T j}\right)+N\left(\sigma_{--}^{T j}\right) \leq N\left(\sigma_{+-}^{T j}\right)+N\left(\sigma_{-+}^{T j}\right), \quad \sigma \in\{-1,1\}^{V^{\prime} \backslash T \cup\{j\}}
$$

where for instance $\sigma_{+-}^{T j}$ denotes the completion of $\sigma$ with +'s on $T$ and - on $j$.

We denote for instance by $C_{T}^{+}(\sigma)$ and $C_{j}^{+}(\sigma)$ respectively the set of +clusters of $\sigma$ adjacent to $T$ and $j$. We have

$$
\begin{aligned}
& N\left(\sigma_{++}^{T j}\right)=N(\sigma)-\left|C_{T}^{+}(\sigma) \cup C_{j}^{+}(\sigma)\right|+1 \\
& N\left(\sigma_{+-}^{T j}\right)=N(\sigma)-\left|C_{T}^{+}(\sigma)\right|+1-\left|C_{j}^{+}(\sigma)\right|+1 \\
& N\left(\sigma_{--}^{T j}\right)=N(\sigma)-\left|C_{T}^{-}(\sigma) \cup C_{j}^{-}(\sigma)\right|+1 \\
& N\left(\sigma_{-+}^{T j}\right)=N(\sigma)-\left|C_{T}^{-}(\sigma)\right|+1-\left|C_{j}^{+}(\sigma)\right|+1
\end{aligned}
$$

The first equation, for instance, uses the fact that if one turns on + 's over $T$ and $j$, the +clusters adjacent to $T$ or to $j$ become an unique cluster. Using in eq. (17) above equations we get

$$
\left|C_{T}^{+}(\sigma) \cap C_{j}^{+}(\sigma)\right|+\left|C_{T}^{-}(\sigma) \cap C_{j}^{-}(\sigma)\right| \leq 2
$$

The left hand side is bounded by the number of vertexes that are simultaneously adjacent to $T$ and $j$. Since $T$ and $j$ are adjacent and $\operatorname{deg}(j) \leq 3$, this number is not greater than 2 . This proves eq. (22) and completes the proof of the proposition.

\section{REFERENCES}

[1] N.L. Biggs, Discrete Mathematics, Oxford Univ. Press (1993).

[2] C. Cammarota, L. Russo, Bernoulli and Gibbs Probabilities of Subgroups of $\{0,1\}^{S}$, Forum Math. 3, 401-414 (1991).

[3] C. Cammarota, On the temperature dependence of the mean number of clusters, Journ. Stat. Phys. 63, 783-790 (1991).

[4] C. Cammarota, Stochastic order and monotonicity in temperature for Gibbs measures, Lett. Math. Phys. 29, 287-295 (1993).

[5] C. Cammarota, Positive and negative correlations for conditional Ising distributions, Rew. Math. Phys. 14, 1099-1113 (2002).

[6] M. Campanino, L. Russo, An upper bond on the critical percolation probability for the three-dimensional cubic lattice, Ann. Prob. 13, 478-491 (1985).

[7] O. Haggstrom, A note on (non-)monotonicity in temperature for the Ising model, Markov Proc. Rel. Fields 2, 529-537 (1996).

[8] G. Grimmett, Percolation, Springer (1999).

[9] G. Grimmett, The random-cluster model in Encyclopaedia of Mathematical sciences: Probability on discrete structures (Kesten Ed.), Springer (2004). 
[10] C. Gruber, A. Hintermann, D. Merlini, Group Analysis of Lattice Systems, Lecture Notes in Physics 60, Springer - Verlag (1977).

[11] H. Meyer-Ortmanns, Functional complexly measure for networks, Physica A337, 679-690 (2004).

Dipartimento di Matematica, Universitá di Roma "La Sapienza" Piazzale A. Moro 2, 00185 Roma, italia.

E-mail address: cammar@mat.uniroma1.it 